\section{Recombinant Human TSH Versus Thyroid Hormone Withdrawal}

TO THE EDITOR: With considerable interest we have read the recent report by Van Nostrand et al. in which the authors compared the proportion of patients with positive scans and the number of lesions detected during diagnostic ${ }^{131}$ I whole-body scintigraphy and ${ }^{124}$ I PET/CT in patients prepared with either recombinant human thyroid-stimulating hormone (rhTSH) or conventional thyroid hormone withdrawal (THW) (1). The authors reported that considerably more patients were "positive" both on diagnostic ${ }^{131}$ I whole-body scintigraphy and on ${ }^{124}$ I PET/CT after THW than after rhTSH. The entire study appears methodologically geared toward comparing ${ }^{131} \mathrm{I}$ with ${ }^{124} \mathrm{I}$. It would therefore have been interesting to see further statistical analyses on the comparison of these 2 wellestablished radioisotopes, especially if the authors additionally would have acquired and evaluated ${ }^{131} \mathrm{I}$ SPECT/CT (2). Instead, the authors performed an analysis of the differences between rhTSH and THW.

Furthermore, the authors surprisingly did not use ${ }^{124}$ I-PET to perform dosimetry for their patients, which in our opinion would have been clinically relevant especially in those patients with metastatic lesions (3) because it is well known that the visualization of metastases does not automatically translate into the possibility of an effective ${ }^{131} \mathrm{I}$ treatment $(4,5)$.

Besides these aspects, we see major drawbacks in the interpretation of the presented data based on the rather weak methodology. The authors already point out in their discussion that patients were not randomized as to the method of preparation, nor was any patient scanned after preparation with both rhTSH and THW. An intraindividual comparison would, however, represent the gold standard for a head-to-head study, especially when taking into account the heterogeneous patient sample. Instead, the decision as to the rhTSH stimulation method was made by referring endocrinologists, whose criteria were subjective, not systematically recorded, and not otherwise reported by Van Nostrand et al. Accordingly, there may well have been a bias between the 2 groups, even though the authors found no statistical difference between the 2 groups regarding age, sex, thyroglobulin level, urine iodine level, type of histology, or indications on the order form. Nonetheless, other variables such as the results of prior tests (e.g., cervical ultrasound) and patient history-including TNM stage (6) - which might have influenced the clinical indication, were not included in the analysis. Of note, all 3 patients with a less favorable prognosis (insular, tall cell, poorly differentiated histology) and a greater likelihood of dedifferentiation were treated in the rhTSH group (12.5\% of that group). Furthermore, the paper does not differentiate whether lesions concerned thyroid remnants or locoregional metastases-assuming that any cervical lesion concerned the latter-whereas the former according to recent literature would have essentially been clinically irrelevant (especially in the context of undetectable thyroglobulin levels). In

COPYRIGHT (C) 2012 by the Society of Nuclear Medicine and Molecular Imaging, Inc. addition, no data are given in which iodine uptake in distant metastases is compared between the 2 methods of stimulation.

The authors state that their result is most consistent with the data of Freudenberg et al. (7) but do not reflect that the conclusion of Freudenberg et al. was far more cautious ("In conclusion, we found some suggestions, but no statistically significant evidence, that rhTSH stimulation may result in a lower radiation dose to advanced [differential thyroid carcinoma] lesions per GBq of administered activity than does THW stimulation.")

A large, multicenter, multinational prospective study (220 patients with evaluable diagnostic scintigraphs) by Haugen et al. performed an intraindividual comparison of preparation with hypothyroidism and rhTSH for diagnostic ${ }^{131}$ I wholebody scintigraphy. The 2 modalities were comparable in their diagnostic yield: with respect to 49 patients with distant metastases, 39 (80\%) were judged by masked independent readers to have concordant diagnostic scans, $2(5 \%)$ to have superior rhTSH scans, and $8(16 \%)$ to have superior THW scans $(P=$ $0.109)(8)$. This finding was crucial for the regulatory approval of rhTSH to prepare patients for diagnostic ${ }^{131} \mathrm{I}$ whole-body scintigraphy.

Given the aforementioned methodologic constraints, the conclusion that "physicians should be cautious in using rhTSH for patient preparation before diagnostic scanning for the detection of [differential thyroid carcinoma]... with ${ }^{131}$ I" seems far too strong, especially when regarding the tremendous burden that THW poses on patients both medically and in terms of decreased quality of life. On the other hand, it is obvious that the therapeutic use of rhTSH in patients with metastases is not covered by the current label in Europe or the United States and must be considered experimental.

As our respected colleagues already elaborate: "A prospective study in which each patient is studied using both methods of preparation is warranted." Although we would very much welcome and would gladly participate in such a study, we would also welcome a more nuanced view on the data presented in the study by Van Nostrand et al.

\section{REFERENCES}

1. Van Nostrand D, Khorjekar GR, O'Neil J, et al. Recombinant human thyroidstimulating hormone versus thyroid hormone withdrawal in the identification of metastasis in differentiated thyroid cancer with ${ }^{131} \mathrm{I}$ planar whole-body imaging and ${ }^{124}$ I PET. $J$ Nucl Med. 2012;53:359-362.

2. Chen L, Luo Q, Shen Y, et al. Incremental value of ${ }^{131} \mathrm{I}$ SPECT/CT in the management of patients with differentiated thyroid carcinoma. J Nucl Med. 2008;49:1952-1957.

3. Lassmann M, Hanscheid H, Verburg FA, Luster M. The use of dosimetry in the treatment of differentiated thyroid cancer. $Q$ J Nucl Med Mol Imaging. 2011; 55:107-115.

4. Chiesa C, Castellani MR, Vellani C, et al. Individualized dosimetry in the management of metastatic differentiated thyroid cancer. $Q \mathrm{~J} \mathrm{Nucl} \mathrm{Med} \mathrm{Mol}$ Imaging. 2009;53:546-561.

5. Freudenberg LS, Jentzen W, Görges R, et al. ${ }^{124}$ I-PET dosimetry in advanced differentiated thyroid cancer: therapeutic impact. Nuklearmedizin. 2007;121-128.

6. Sobin LH, Gospodarowicz MK, Wittekind C. TNM Classification of Malignant Tumours. 7th ed. New York, NY: Wiley-Blackwell; 2009.

7. Freudenberg LS, Jentzen W, Petrich T, et al. Lesion dose in differentiated thyroid carcinoma metastases after rhTSH or thyroid hormone withdrawal: ${ }^{124} \mathrm{I}$ PET/CT dosimetric comparisons. Eur J Nucl Med Mol Imaging. 2010;37:2267-2276. 
8. Haugen BR, Pacini F, Reiners C, et al. A comparison of recombinant human thyrotropin and thyroid hormone withdrawal for the detection of thyroid remnant or cancer. J Clin Endocrinol Metab. 1999;84:3877-3885.

\section{Frederik A. Verburg* \\ Markus Dietlein \\ Lutz Freudenberg \\ Sophie Leboulleux \\ Fabian Pitoia \\ Christoph Reiners \\ Marcel Stokkel \\ Markus Luster \\ *University Hospital Aachen \\ Pauwelsstrasse 30 \\ 52074 Aachen, Germany \\ E-mail: fverburg@ukaachen.de}

Published online Aug. 30, 2012.

DOI: 10.2967/jnumed.112.108738

REPLY: We always welcome a professional dialogue from esteemed colleagues in regard to studies that we have published, and we believe that such a dialogue helps us all move forward to a more accurate understanding of the world about us. Continuing in that spirit, I would like to address the various comments in the letter to the editor from Verburg et al. regarding our report comparing the number of metastatic lesions of differentiated thyroid cancer (DTC) detected after preparation with thyroid hormone withdrawal (THW) versus injections of recombinant thyroid-stimulating hormone (rhTSH) (1).

Our understanding of the main point of their letter is that they believe that our recommendation regarding selective use of rhTSH is too strong and that they would like to see a more "nuanced" view on the data presented. Our recommendation was that, until more data become available, physicians should be cautious in using rhTSH for patient preparation before diagnostic scanning for the detection of DTC or treatment of distant metastases secondary to DTC with ${ }^{131} \mathrm{I}$. Insofar as the data support this recommendation, it would not appear appropriate to characterize the recommendation as being too strong. When data are clear-cut, there is less accommodation for "nuance."

With regard to the observation of Verburg et al. that our entire study appeared methodologically geared toward comparing ${ }^{131} \mathrm{I}$ with ${ }^{124}$ I-indeed, the data were obtained from our previously published original study (2) comparing ${ }^{131} \mathrm{I}$ planar imaging with ${ }^{124} \mathrm{I}$ PET, with 16 additional patients studied and included. However, whether the data were derived from a study comparing lesion detection of ${ }^{131} \mathrm{I}$ planar imaging with ${ }^{124}$ I PET is not in and of itself a limitation. Although there were limitations to our study that we recognized and discussed in the publication, we do not believe this is one of them.

With regard to the interest of Verburg et al. in seeing further statistical analyses comparing the 2 radioisotopes, especially if we additionally had acquired and evaluated ${ }^{131}$ I SPECT/CT, we noted in our original publication (2) that a comparison of ${ }^{131}$ I SPECT/CT with ${ }^{124}$ I PET would have been valuable for that study. However, such a comparison was not critical to the present study (1) because we were comparing planar imaging with planar imaging and PET with PET. To address Verburg et al.'s further opinion that we analyzed differences between rhTSH and THW instead of comparing lesion detection on ${ }^{131} \mathrm{I}$ planar imaging versus ${ }^{124} \mathrm{I}$ PET, we did not perform evaluations after rhTSH and THW instead of lesion detection but in addition to lesion detection.

Verburg et al. subsequently state that they were surprised we did not use ${ }^{124}$ I-PET to perform dosimetry for our patients. They believe this would have been clinically relevant, especially in patients with metastatic lesions, because visualization of metastases does not automatically indicate the possibility of an effective ${ }^{131}$ I treatment. Several important facts will help clarify this issue. First, the calculated radiation absorbed dose to a focal lesion or organ as determined by the various methods of ${ }^{124}$ I dosimetry does not necessarily correlate with clinical outcomes or side effects $(3,4)$. However, we do agree that lesional dosimetry should be performed - not necessarily to indicate clinical relevance based on a calculated radiation absorbed dose but rather to indicate clinical relevance based on a comparison of relative lesional radiopharmacokinetics. We have such a study already under way, as well as another study comparing ${ }^{124}$ I dosimetry after preparation with THW and rhTSH injections in patients serving as their own controls. Nevertheless, because clinical outcomes are more important as an endpoint than the calculated radiation absorbed dose by ${ }^{124} \mathrm{I}$ dosimetry, our paper (1) referred to work from our institution by Klubo-Gwiezdzinska et al., who demonstrated no difference in outcomes when patients with metastatic DTC were prepared for ${ }^{131}$ I treatment with either rhTSH or THW (5). Although THW scans may allow better detection of metastatic lesions than do rhTSH scans, preparation with THW may not necessarily result in significantly more radiation absorbed dose to the metastases than does preparation with rhTSH, thereby not improving outcomes. Thus, the caveat implied by Verburg et al. in regard to the lack of lesional dosimetry using ${ }^{124}$ I does not mitigate the fact that more lesions were detected after preparation with THW versus rhTSH injections and that - as concluded in our paper-until more data become available, physicians should be cautious in using rhTSH for patient preparation before diagnostic scanning for the detection of DTC or treatment of distant metastases secondary to DTC with ${ }^{131} \mathrm{I}$.

In drawing attention to methodology-based drawbacks in our interpretation of the presented data, Verburg et al. are simply repeating limitations of our study that we already noted in our discussion.

Next, Verburg et al. note that our statement that our result was most consistent with the data of Freudenberg et al. did not reflect the fact that the conclusion of Freudenberg et al. was more cautious. Our actual statement was, "In comparing our data with other reports that evaluated preparation with THW and rhTSH, our data are most consistent with those of Freudenberg et al. (6).... .In the study of Freudenberg et al. (6), their endpoint was the estimation of the radiation absorbed dose to the metastatic foci after THW and rhTSH preparation. They reported that the mean radiation absorbed dose for the lesions identified in a group of patients $(n=27)$ prepared with rhTSH was only $60 \%$ of the radiation absorbed dose to lesions in another group of patients $(n=36)$ prepared with THW. However, this difference was not statistically different." I will leave the judgment to the reader regarding whether our statement reflected the data and conclusion of Freudenberg et al. and whether our data are most consistent with their data.

Interestingly, Verburg et al. reference an article by Haugen et al. (7) as evidence that patient preparation with rhTSH injections has already been shown to be equivalent to patient preparation with THW. However, Verburg et al. do not point out the limitations of the study by Haugen et al. Notably, Haugen et al. reported that THW scans were superior to rhTSH scans in $16 \%(8 / 49)$ of patients, albeit not to a statistically significant extent $(P=0.109)$. Second, although Verburg et al. state that this information was crucial for the approval of rhTSH (Thyrogen; Genzyme Corp.) by the Food and Drug Administration, it has not approved Thyrogen for use in metastatic DTC in the United States, which is stated in the drug insert. Third, the order of THW and rhTSH scans was not randomized; all rhTSH scans were performed first. Although Haugen et al. recog- 